\title{
Et si, au Louvre, on respirait du fantôme ! Géocritique d'un espace muséal
}

\author{
And If, at the Louvre, We Breathed a Ghost! \\ Geocriticism of a Museum As a Space
}

\begin{abstract}
RÉSUMÉ
Les fantômes du Louvre d'Enki Bilal met en scène une exposition de cet artiste qui s'est déroulée dans ce même lieu. L'auteur propose une visite inhabituelle du musée : il a choisi des œuvres à partir desquelles il a fait resurgir autant de fantômes. L'artiste a superposé les fantômes aux photographies et a construit une biographie pour chaque protagoniste. En recourant à la géocritique, nous tenterons de répondre aux questions suivantes : comment l'auteur s'empare-t-il du musée pour construire des mondes parallèles multiples et hétérogènes ? Comment le musée devient-il à la fois un lieu de terreur et d'admiration ?
\end{abstract}

Mots-clés : musée, fantôme, art, géocritique, littérature, Louvre

\section{ABSTRACT}

The Ghosts of the Louvre by Enki Bilal embodies an exhibition of this artist which took place in this same place. The author offers an unusual visit to the museum: he has chosen works from which he brings up so many ghosts. The artist juxtaposed the ghosts and the photographs taken and built a biography for each protagonist. Using geocriticism, we will answer the following questions : how does the author take over the museum to build multiple and heterogeneous parallel worlds? How does the museum become both a place of terror and of admiration?

Keywords: museum, ghost, art, geocriticism, literature, Louvre

Avant d'entreprendre l'analyse du livre Les fantômes du Louvre, nous allons préciser les fondements théoriques de notre travail. Il est nécessaire de souligner que la finalité de notre démarche est de mettre en évidence les enjeux et les interactions des univers de la réalité et de la fiction par le biais de la géocritique. Cette dernière, que nous allons appliquer à l'étude du musée du Louvre, revalorise non seulement l'étude de l'espace, mais insiste aussi sur sa complexité, en mettant l'accent sur son aspect flottant, hétérogène et multiple. Pour cela, le choix des concepts, celui d'abord des mondes parallèles, que nous précisons dans la première

\footnotetext{
Lamia Mecheri, Department of French, Faculty of Literature, Social Sciences and Humanities, Badji Mokhtar University of Annaba, PB 12, 23000 Annaba, lamiarome@yahoo.fr, https://orcid.org/0000-0001-9570-3224
} 
partie - intitulée le « Louvre-monde ou les mondes parallèles »-et, ensuite, celui de l'encore-vide, qui est développé dans la seconde - intitulée « Spectacle vivant et musée »-, dévide notre fil d'Ariane que justifie la logique de notre pensée, divisant notre analyse en deux parties successives. Le premier concept nous permet de poser les fondements du lieu muséal, afin d'examiner ses représentations, en le déconstruisant ou mieux en le fictionnalisant. Le but est de mettre en valeur les interactions de l'espace réel et de l'espace fictionnel à partir desquels émerge un univers symbolique mouvant et changeant où les frontières sont sans cesse remises en cause. L'aspect mobile du lieu nous conduit systématiquement à mettre en œuvre le second concept. Ce dernier nous permet d'explorer le Louvre à travers les époques, puisque l'étude de l'espace, en géocritique, ne peut être détachée de celle du temps, qui tend à se spatialiser, en franchissant des frontières perméables qui sont, non seulement, spatiales mais aussi temporelles.

\section{Le Louvre-monde ou les mondes parallèles}

Dans cette première partie, nous empruntons le concept des mondes parallèles, initié par Bertrand Westphal. Ce dernier, dans l'article intitulé « Parallèles, mondes parallèles, archipels », propose une définition du mot « parallèle », en partant du domaine de la géométrie euclidienne : «En géométrie euclidienne, deux lignes sont dites parallèles lorsqu'elles ne se rencontrent pas » (Westphal, 2001, p. 235). Il propose une autre définition géographique : « le parallèle est un cercle conservant une égale proportion de distance à l'égard du plan de l'Équateur » (p. 235). Mais, pris dans un contexte littéraire et donc métaphorique, le parallèle littéraire « $[\ldots]$ n'est pas foncièrement aporétique. Si les droites restent équidistantes, c'est l'espace qui se déploie entre elles? s'il n'est pas vide ? qui établit la relation » (p. 236). Dans cette approche, ce qui nous intéresse est le mot « relation», non d'un espace ordinaire, mais celle d'une espèce d'espaces, lorsqu'ils sont soumis aux lois de la géocritique. En outre, cette dernière ne se contente pas uniquement de réfléchir sur la relation qui existe entre les espaces. Au contraire, elle se charge d'examiner leurs interactions. L'espace se multiplie et, à son tour, devient métaphorique dans la mesure où il est question d'un monde fictionnel qui interagit avec un monde réel. De cette manière, les mondes parallèles prennent forme au sein de la littérature. Cette dernière, favorable au discours sur le parallèle en raison de son hétérogénéité, est « [...] à l'intersection; elle figure en un centre abstrait, toujours mobile, ponctuel, qui fédère toutes les tensions concrètes » (p. 241). Le concept des mondes parallèles nous fait penser, bien évidemment, à la théorie des mondes possibles de Thomas Pavel, une théorie qui inspire entre autres la géocritique et qui, elle-même, a été élaborée par des penseurs à l'instar de Leibniz et de Deleuze. Elle postule l'hypothèse selon laquelle il existe d'autres mondes que le nôtre. Dans le domaine littéraire, ce sont des mondes parallèles dont les frontières bougent et interagissent de façon constante. 
En appliquant le concept des mondes parallèles au livre Les fantômes du Louvre, nous remarquons qu'Enki Bilal a choisi de situer les différentes histoires des fantômes qu'il a créés au sein du musée du Louvre, comme l'annonce le titre de l'ouvrage. Le choix du Louvre n'est pas anodin puisque, à l'évidence, il est l'un des plus célèbres musées du monde, en raison de sa localisation géographique et de sa réputation culturelle mondiale. Rappelons qu'il s'est inséré dans le palais royal, depuis le départ de Louis XIV et son installation, en 1682, au château de Versailles. Le musée est doté d'une longue et riche histoire et renferme des trésors précieux, comme les œuvres d'art et des objets de collection inestimables, qui attirent les touristes et les spécialistes en art, en architecture, en géographie, en sociologie, etc. En outre, il est situé au cœur de la ville des Lumières, connue pour son aspect culturel et artistique, mais aussi à travers les innombrables descriptions des auteurs, comme Charles Baudelaire ou encore Émile Zola. Au fil du temps, un lien solide entre le Louvre et Paris, s'est établi et consolidé en instaurant un dialogue mutuel culturel, historique, artistique, etc. -, comme l'explicite le passage suivant :

Inscrit dans le Palais des rois de France, au cœur du centre historique et politique de Paris, le musée du Louvre s'est transformé au fil des siècles sous les effets consécutifs d'une urbanisation et métropolisation grandissantes et d'une fréquentation touristique mondialisée auxquelles s'est jointe la forte attente culturelle généralisée des citadins pour les musées (Krebs, 2016).

Pour toutes ces raisons, le Louvre continue d'être une source d'inspiration inépuisable pour les artistes. Enki Bilal n'échappe pas à la règle. En fait, l'artiste revisite le musée de façon inédite puisque, dans son livre, il devient un lieu métaphorique. En réalité, il est question de deux espaces muséaux, l'un est géographique et historique et donc réel. Quant à l'autre, il est fictif. Dans tous les cas, l'auteur, qui entretient une relation particulière avec le Louvre, construit sa propre géographie du lieu, une géographie symbolique et imaginaire, qui nous permet d'y repérer une seconde représentation des lieux réels du musée. Il pose un cadre délimité dans lequel la réalité du musée peut se matérialiser. L'ancrage de l'espace réel dans la fiction relève, bien évidemment, de la créativité et du talent de l'auteur. Ce dernier, à travers la représentation du Louvre, laisse transparaître son monde intérieur. Il intériorise l'espace muséal en décrivant les œuvres qui le fascinent et les endroits visités qui captent son attention, selon les besoins de la narration. C'est dire l'importance et la préoccupation qu'accorde Bilal à la construction de son espace fictionnel et à la manière de faire ressurgir les fantômes qui hantent les couloirs du musée, à partir des œuvres sélectionnées.

L'auteur entreprend une démarche particulière en convoquant les fantômes au sein de l'établissement artistique. Il réinvente le réel muséal à travers son propre regard, en créant une sorte de " géographie parallèle » ${ }^{1}$ des lieux et en proposant

\footnotetext{
1 Cette expression nous renvoie à l'article d'Nkunzimana (2018, pp. 29-45).
} 
une vision originale des œuvres célèbres exposées au Louvre. Il affirme avoir passé des heures entières au musée, surtout le jour de sa fermeture, où il y a le moins de monde, pour être en contact privilégié avec les œuvres. Ainsi, il a endossé le rôle de chasseur d'œuvres d'art qui l'ont intéressé, afin de faire apparaître un fantôme auquel il ne s'attend pas, puisque lui-même ne sait pas quel type de fantôme allait ressurgir de la prise de telle ou telle photo ; il était en état de découverte et d'impatience. Les lecteurs du livre ou les visiteurs de l'exposition, ayant eu lieu au Louvre du 20 décembre 2012 au 18 mars 2013, découvrent, par exemple, que le fantôme qui hante la statue de la Victoire de Samothrace se nomme Aloyisias Alevratos. L'habitant de la stèle du Code de Hammurabi, roi de Babylone porte le nom d'Enheduana Arwi-a. Quant au rodeur près de La Joconde, il est connu sous le nom d'Antonio di Aquila, etc. Mais, avant de libérer ces esprits, l'artiste explique son approche, une attitude qui s'approprie les caractéristiques de la littérature en termes d'inachèvement, puisqu'il compte recommencer son travail artistique à chaque fois qu'il visite le musée, afin de rencontrer de nouveaux fantômes et d'en livrer de nouvelles représentations et interprétations des lieux. D'ailleurs, dans la préface de son livre, il explique :

J'ai dû faire près de quatre cents photos, dans des périodes de musée désert, rare moment de privilège.

Quatre cents photos, c'est peu. Il y avait donc du tri, déjà, inconscient, peut-être influencé par « eux ».

De grandes œuvres emblématiques sont passées à la trappe. Peut-être leurs fantômes étaient-ils médiocres ? Ou peut-être l'ai-je été moi... Car des regrets, j'en ai.

Et chaque fois que je remettrai les pieds dans ce vivier magnifique, d'une manière ou d'une autre, je traquerai les manquants [guillemets de l'auteur cité] (Bilal, 2012, p. 3).

La fin de la citation nous montre clairement que les arts impactent les lieux en leur procurant un caractère mouvant. Il est question ici d'un espace ouvert et fluide qui échappe à La cage des méridiens, pour reprendre le titre métaphorique de Westphal, ouvrage dans lequel l'auteur remet en cause la notion de « limite », en questionnant les relations entre la littérature et l'art contemporain : « Idéalement, l'art permet de promouvoir l'ouverture de l'espace au détriment de la clôture du lieu. [...] La performance esthétique à laquelle se livrent l'art et le discours qui lui est associé n'est pas séparé du monde » (Westphal, 2016, pp. 261-262), écrit le géocritique. En ce sens, dans cet espace métaphorique, provisoire puisque changeant en raison de sa mobilité, le monde de la fiction entre en connexion avec le monde réel. Ces deux univers deviennent, au sens de Westphal, des mondes parallèles qui interagissent au sein d'un espace hétérogène. Ainsi, les mondes parallèles ne permettent plus seulement aux lecteurs de repérer les espaces muséaux tel que représentés par Enki Bilal, mais de les traverser et de les vivre différemment. Ils les invitent à les déchiffrer et à les lire vraiment, comme le 
suggère la citation de Perec, reprise par le géocritique : «Bref, les espaces se sont multipliés, morcelés et diversifiés. [...] Vivre, c'est passer d'un espace à un autre, en essayant le plus possible de ne pas se cogner $»^{2}$ (Perec, 1974, p. 14).

Outre les fantômes qui nous permettent de lire les espaces et d'assurer la jonction entre les mondes parallèles, l'artiste se sert de l'architecture du musée en décrivant des espaces à sa manière, c'est-à-dire en convoquant des fantômes. C'est, pour lui, une autre façon de déconstruire le Louvre et de le reconstruire en l'insérant dans la fiction. En fait, lors de ses déplacements, au sein de l'établissement, Enki Bilal attire l'attention sur des éléments architecturaux, faisant partie de l'espace muséal stratifié, qui l'ont impressionné en les fictionnalisant. En ce sens, il est question des « Salles Rouges » gardées par le fantôme Djeynaba :

Salles Daru et Mollien, qui ont été décorées par Alexandre Dominique Denuelle pour le musée impérial en 1863, comme l'indique leur décor rouge et or. Elles abritent aujourd'hui les peintures de grands formats de l'école romantique française (salle Mollien) et du néoclassicisme français (salle Daru) [parenthèses de l'auteur cité] (Bilal, 2012, p. 44).

Puis, au fil de la lecture, nous découvrons un autre fantôme, Colonel Markus Dudke, qui se promène dans la Grande Galerie, construite entre 1595 et 1610. Cette dernière permettait de relier le château du Louvre au palais des Tuileries : « Elle (la Grande Galerie) fut utilisée au XVII siècle pour la cérémonie des écrouelles, durant laquelle le roi guérissait les malades par imposition des mains » [parenthèses de l'auteur cité] (Bilal, 2012, p. 50), écrit l'artiste.

Enfin, Bilal nous permet de rencontrer Lyubino Nuzri, un fantôme qui rôde dans la Chambre à Alcôve, construite par les architectes Claude Perrault, Louis le Vau et François d'Orbay entre 1668 et 1678, comme l'explique l'artiste :

Elle (la Chambre à Alcôve) tire son nom des boiseries mises en place en 1831 par l'architecte Fontaine. Les éléments, sculptés en 1654, proviennent de la chambre à coucher, aménagée par l'architecte Le Vau pour Louis XIV, autrefois située au premier étage du pavillon du Roi, et transformée en 1817 [parenthèses de l'auteur cité] (Bilal, 2012, p. 116).

L'évocation de l'architecture nous permet de déceler de nouveaux mondes parallèles en rapport direct avec l'art et qui, eux-mêmes, fonctionnant comme une mise en abyme dans un langage littéraire, font partie des mondes parallèles relatifs à l'espace muséal, où s'entrecroisent réel et imaginaire. L'architecture, la sculpture, la photographie, la littérature et la peinture sont ces mondes parallèles, typiquement artistiques qui émanent de l'univers muséal. Ils sont pour l'auteur un moyen de cartographier de nouveaux paysages, des paysages qui, à priori, sont mentaux et, aussi, fluides puisque aléatoires.

\footnotetext{
2 Cette citation est très utilisée en géocritique, précisément dans l'article de Westphal (2000, p. 11).
} 
En effet, à travers les œuvres artistiques examinées par Enki Bilal, nous constatons que ces mondes parallèles artistiques mettent en scène des univers différents en convoquant des lieux mythiques, d'Orient et d'Occident, qui continuent à vivre à travers les âges. Citons, à titre d'exemple, le buste nommé Tête d'homme - exposé au département des Antiquités égyptiennes et habité par le fantôme Ahmosé Chepseset -, le tableau de la Jeune Orpheline au cimetière exposé au département des Peintures et hanté par Lantelme Fouache -, le Casque de type corinthien - exposé au département des Antiquités grecques, étrusques et romaines où rôde Hécube, le Taureau androcéphale ailé mésopotamien, exposé au département des Antiquités orientales où erre Lakshek -, etc. Ainsi, la diversité des lieux et les origines des œuvres d'art procurent au Louvre un caractère universel. C'est pourquoi le musée, ou mieux le «Louvre-monde », se transforme en un espace frontalier qui se raconte et qui, non seulement, fait dialoguer les lieux, mais aussi les époques. Il devient l'Atlas des égarements par excellence, pour reprendre la première partie du titre du dernier ouvrage de Westphal, « [...] car c'est à la faveur des égarements que poindra la nouveauté » (Westphal, 2019, p. 13), souligne le géocritique.

Cependant, il ne faut pas oublier de souligner qu'à l'analyse des espaces se superpose celle du temps. Ce dernier, comme l'espace, se stratifie. En effet, le facteur temporel ne peut être détaché du vecteur spatial, ce que nous allons étudier dans la seconde partie à travers les portraits de certains fantômes.

\section{Spectacle vivant et musée}

Dans cette partie, nous allons nous servir du concept d'encore-vide, à travers les fantômes errants dans le Louvre, afin de mettre en lumière l'espace muséal et les émotions qu'il suscite. La « Représentation de l'encore-vide », par opposition à la «Représentation du trop-plein », est une partie du titre de l'ouvrage La géocritique. Réel, fiction, espace de Bertrand Westphal. Le concept, en relation avec l'étude de la carte géographique et imaginaire, a été créé dans un contexte postmoderne, ayant un enjeu colonial et postcolonial. En ce sens, l'homme conquérant est souvent confronté à un dilemme : soit il remplit des espaces vides, soit il vide des espaces trop pleins, comme le précise le géocritique : « Horreur! Quand tout se remplit, il faut refaire de la place» (Westphal, 2007, p. 100). Ce sont là les caractéristiques de l'homme postmoderne qui « [...] ne cesse d'être confronté au sentiment d'un remplissage universel » (p. 100). De ce fait, la littérature se transforme en un laboratoire du « possible » permettant à l'artiste de mettre en œuvre ses fantasmes territoriaux : «La littérature, comme toutes les formes d'art mimétique, devient dans ce contexte le champ d'expérimentation de réalités alternatives, qui visent à redonner de la marge à l'imaginaire et à ce qui l'alimente : le référent » (p. 100).

Pour revenir à notre contexte, partons de l'hypothèse que le musée est un espace encore-vide, dans la mesure où Enki Bilal donne vie aux œuvres qui y sont 
exposées, à travers son travail artistique. Il remplit l'espace muséal en le peuplant de fantômes errants. Il est vrai que l'artiste ne ranime pas toutes les œuvres d'art puisque, en fin de compte, il choisit vingt-deux éléments parmi lesquels on retrouve des tableaux, des sculptures, des artefacts et des éléments architecturaux. L'on s'interroge alors pourquoi l'artiste a-t-il sélectionné précisément vingt-deux œuvres? Et pourquoi certaines œuvres et pas d'autres ? À ce sujet, l'auteur nous livre une réponse « artistique », émaillée de doute, en expliquant :

Pourquoi vingt-deux, et pas un de plus ou de moins, et pourquoi, surtout, ceux-là et pas d'autres, beaucoup d'autres possible. Pas de réponse... Ou alors, c'est peut-être ces vingt-deux-là qui ont fait signe, qui ont voulu plus que les autres, qui ont joué des coudes, se sont imposés, faisant davantage corps avec leur œuvre, leur espace. L'ambition chez les fantômes existerait donc (Bilal, 2012, p. 3).

Dans cet extrait, nous remarquons que, au sein de cet espace métaphorique où tout devient possible, l'auteur entre en contact avec les fantômes qui se sont présentés à lui. Les frontières du Louvre deviennent perméables et permettent aux sujets de se déplacer d'un espace à un autre et aussi d'une époque à une autre. Dans un discours narratologique et littéraire, ce procédé, où l'artiste communique avec ses propres personnages, se nomme une métalepse, un concept créé par Gérard Genette. Bilal rencontre non seulement ses personnages, mais aussi anticipe leur façon d'interpréter son travail artistique. Ainsi, l'auteur s'interroge sur le statut du fantôme et ne cherche nullement à démythifier les œuvres exposées, c'est-à-dire à leur ôter leur caractère mythique dans la mesure où elles subsistent à travers les époques et résistent au « Temps ». Au contraire, pris d'admiration pour ces merveilles, il recrée un dialogue entre des œuvres majeures, en leur rendant hommage ainsi qu'à leurs artistes, témoins de leur temps.

De ce point de vue, la fiction, ou mieux la science-fiction qui nourrit profondément les textes d'Enki Bilal, devient une voie ayant le pouvoir de tisser un lien entre les époques et les lieux, comme l'affirme l'auteur, lors d'une interview :

Oui. Dans mes créations, les mondes passé, présent et futur sont toujours intimement liés. Tout ce que je fais est tiré de ce magma temporel, ma nourriture est là. J'ai le sentiment de coller à l'époque, et même de la devancer avec des fulgurances qui sont un peu le propre de tout artiste (Potet, 2017).

Toutefois, avant de créer les personnages-fantômes, Enki Bilal s'est d'abord renseigné sur l'époque de la création des œuvres exposées qui l'ont attiré au Louvre. Puis, il a inventé des biographies pour ses personnages qui, au début, lui étaient inconnus, qu'il a fait croiser avec des éléments historiques existant et ayant capté son attention. Ainsi, l'auteur invente et improvise des vies en partant d'un élément concret, avec un arrière-plan historique. Il s'est appuyé sur des détails de 
la vie réelle de chaque époque, représentée à travers les vingt-deux œuvres, pour fabriquer des personnages fictifs. Ces derniers portent des noms et des identités multiples, comme nous l'avons mentionné dans la première partie. Or, l'examen de chaque portait des protagonistes nous fait découvrir que ceux-là décèdent dans des conditions violentes, ce qui donne place à l'apparition fantomatique.

Bilal reprend, à l'évidence, des histoires de fantômes liées à la croyance populaire, prétendant qu'un spectre fait une apparition « surnaturelle » lorsqu'une personne décède de façon violente, comme le voudrait la tradition. Cela a, bien sûr, donné naissance à une littérature abondante sur la thématique du fantôme et de ses dérivés - comme les revenants, les spectres, les ombres, etc.-, qu'il serait vain de cerner. Ainsi, les personnages se transforment en fantômes que l'artiste convoque au sein du Louvre, des fantômes qui n'appartiennent ni à notre époque, ni au monde réel. Ils rôdent dans un espace encore-vide et métaphorique, à la croisée $\mathrm{du}$ réel et de la fiction. Dès lors, la mort violente de ces personnages devient le fil conducteur dans le livre Les fantômes du Louvre. En ce sens, l'auteur crée des personnages qui ont vécu des destins proches et les adapte chacun à son époque. Cela donne l'impression que ces personnages-fantômes, empreints de réalisme, ont réellement existé, ce qui renforce le caractère plausible des œuvres et des fantômes qui les accompagnent, issus de différents lieux et époques. On découvre, tour à tour, que le personnage Aloyisias Alevratos (fantôme de la Victoire de Samothrace) est mort dans un accident, " Main droite et tête sont tranchées sur le coup » (Bilal, 2012, p. 9). Le corps inanimé d'Antonio di Aquila (La Joconde) baigne dans son sang, «[...] au pied de la Joconde toujours inachevée » (p. 15). Enheduana Arwi-A meurt lors d'un tremblement de terre, «[...] son corps est découvert dans un temple, écrasé sous les pierres » (p. 21), etc.

En fait, l'auteur établit un lien historique et imaginaire en donnant naissance à des personnages qui, à un moment donné, rencontrent l'œuvre exposée. Parlà, il réinstaure un lien entre lui et les œuvres artistiques qui, jusqu'à nos jours, continuent de fasciner les générations et participent à la transmission de la culture universelle, puisqu'elles appartiennent à la mémoire collective. D'ailleurs, la mémoire est une thématique importante dans l'œuvre d'Enki Bilal : « Tout artiste est forcément confronté à la question de la mémoire, même ceux dont le domaine est l'autofiction. [...] j'ai toujours été sensible à la mémoire collective, que je compare à un air qu'on respire » (Potet, 2017), dit-il. Cela lui permet de voir les œuvres autrement, c'est-à-dire de les voir sous un nouvel angle, en offrant aux lecteurs une nouvelle interprétation de chaque pièce choisie. La présence fantomatique procure au musée un aspect mobile et vivant par le biais des œuvres artistiques, comme si le spectateur assistait à un spectacle vivant, inspirant à la fois terreur et admiration.

Les fantômes font voyager les visiteurs à travers un espace-temps symbolique, où présent passé et futur sont mélangés en enjambant les époques et les lieux, et 
font ressortir leurs sentiments et vibrations lorsqu'ils se promènent dans l'espace muséal encore-vide. Les visiteurs marchent sur les traces de l'auteur en tentant de déchiffrer son œuvre fictionnelle et de dégager les émotions qui en émanent. C'est pourquoi certaines demeurent plus sombres que d'autres, selon l'époque, peut-être même méconnus du grand public, et suscitent la terreur, comme Le Bøuf écorché (1655) de Rembrandt près duquel erre le fantôme Willem Tumpeldt. D'autres, au contraire, sont devenues des pièces célèbres, voire même des icônes du musée, provoquant l'émerveillement comme La Joconde (entre 1503 et 1506) de Léonard De Vinci, près de laquelle rôde Antonio di Aquila, que nous avons mentionné précédemment. Cette toile emblématique, qui renferme plusieurs messages codés, ne cesse de susciter le mystère qui l'entoure, les mythes qui se sont construits autour d'elle, au fil du temps, et les théories qui en découlent, par rapport à son identité, à son sourire, à son regard, à son esthétique, etc. De cette façon, le Louvre, espace paradoxal - vécu entre admiration et mépris -, se transforme en un lieu mythique proposant aux spectateurs une expérience esthétique inédite : «C'est tout le défi des musées, car, ne l'oublions pas, le grand public ne vient pas lire les documents exposés, mais se confronter esthétiquement avec eux » (Sanchez, 2016), souligne Cecilia Sanchez. Enfin, terminons notre analyse de l'espace muséal par une citation de notre auteur, qui nous projette au « Louvremonde », en anticipant les émotions ressenties lors du voyage spatio-temporel à l'issue de la visite de l'établissement artistique :

C'est comme si au Louvre on respirait du fantôme. À chaque coin de galerie, dans chaque parcelle d'œuvre, dans tout ce que les yeux touchent, partout, dans et sur le parquet, dans les replis des murs, dans tout l'air qui colle aux plafonds... En sortant du musée, on en recracherait des bribes immiscées dans les poumons le temps de la visite, du côté Rivoli ou sur les quais de Seine, bribes qui s'en retourneraient aussitôt à leur place, comme aspirées par leur destin, inamovibles témoins scellés à leur temps (Bilal, 2012, p. 3).

\section{References}

Bilal, E. (2012). Les fantômes du Louvre. Paris: Musée du Louvre Éditions.

Louvre. RetrievedFebruary6,2020, fromhttps:/www.louvre.fr/le-louvre-monde-un-lieu-des-territoires-0. Nkunzimana, O. (2018). Pour une géographie parallèle : essai sur le film „Un été à la goulette” de Boughédir. In M. Diouf, \& F. Naudillon (Eds.), Spatialités littéraires et filmiques francophones : nouvelles perspectives (pp. 29-45). Montréal: Éditions Mémoire d'encrier.

Perec, G. (1974). Espèces d'espaces. Paris: Galilée.

Potet, F. (2017, 30 May). L'autofiction sociétale ne m'intéresse pas. Le Monde. Retrieved February 15, 2020, from https://www.lemonde.fr/m-perso/article/2017/05/30/enki-bilal-il-ne-fautjamais-mariner-dans-le-milieu-dans-lequel-on-cree_5135812_4497916.html.

Sanchez, C. (2016). Phénomène : Quand les écrivains investissent les musées. Book.Node. Retrieved February 16, 2020, from https://booknode.com/actus-litteraires/2016/05/10/phenomeneecrivains-investissent-musees/.

Westphal, B. (2000). Pour une approche géocritique des textes - Esquisse. La Géocritique : mode d'emploi, 9-40. Retrieved February 6, 2020, from https://sflgc.org/bibliotheque/ westphal-bertrand-pour-une-approche-geocritique-des-textes/. 
Westphal, B. (2001). Parallèles, mondes parallèles, archipels. Revue de littérature comparée, 298, 235-241.

Westphal, B. (2007). La Géocritique. Réel, fiction, espace. Paris: Minuit.

Westphal, B. (2016). La Cage des Méridiens. La littérature et l'art contemporain face à la globalisation. Paris: Minuit.

Westphal, B. (2019). Atlas des égarements. Études géocritiques. Paris: Minuit. 